\title{
Novel methicillin-resistant coagulase-negative Staphylococcus clone isolated from patients with haematological diseases at the Blood Bank Centre of Amazon, Brazil
}

\author{
Cristina Motta Ferreira' ${ }^{1,2} /{ }^{+}$, Felipe Gomes Naveca ${ }^{3}$, William Antunes Ferreira ${ }^{4}$, \\ Cíntia Mara Costa de Oliveira ${ }^{5}$, Maria das Graças Vale Barbosa ${ }^{2,4}$ \\ ${ }^{1}$ Fundação Estadual de Hematologia e Hemoterapia do Amazonas, Manaus, AM, Brasil ${ }^{2}$ Universidade do Estado do Amazonas, Manaus,

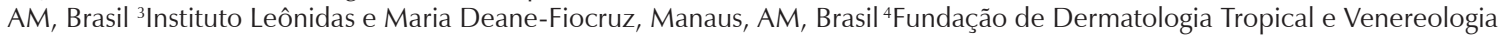 \\ Alfredo da Matta, Manaus, AM, Brasil ${ }^{5}$ Fundação de Medicina Tropical Dr Heitor Vieira Dourado, Manaus, AM, Brasil
}

Methicillin-resistant Staphylococcus remains a severe public health problem worldwide. This research was intended to identify the presence of methicillin-resistant coagulase-negative staphylococci clones and their staphylococcal cassette chromosome mec (SCCmec)-type isolate from patients with haematologic diseases presenting bacterial infections who were treated at the Blood Bank of the state of Amazonas in Brazil. Phenotypic and genotypic tests, such as SCCmec types and multilocus sequence typing (MLST), were developed to detect and characterise methicillin-resistant isolates. A total of 26 Gram-positive bacteria were isolated, such as: Staphylococcus epidermidis (8/27), Staphylococcus intermedius (4/27) and Staphylococcus aureus (4/27). Ten methicillin-resistant staphylococcal isolates were identified. MLST revealed three different sequence types: S. aureus ST243, S. epidermidis ST2 and a new clone of S. epidermidis, ST365. These findings reinforce the potential of dissemination presented by multiresistant Staphylococcus and they suggest the introduction of monitoring actions to reduce the spread of pathogenic clonal lineages of $\mathrm{S}$. aureus and $\mathrm{S}$. epidermidis to avoid hospital infections and mortality risks.

Key words: SCCmec - ST clones - coagulase-negative Staphylococcus

Methicillin-resistant staphylococci stand as main pathogens responsible for high levels of infectious processes worldwide. Methicillin resistance is encoded by the mecA gene, located at the staphylococcal cassette chromosome mec (SCCmec). In Staphylococcus aureus, types I, VII and XI are recognised, whereas types IV and $\mathrm{V}$ are found in Staphylococcus epidermidis and Staphylococcus haemolyticus (Strandén et al. 2009, Iorio et al. 2012). The genotype distributions of staphylococci differ across different geographical regions. The USA300 community-acquired methicillin-resistant $S$. aureus clone sequence typing ST8 is dominant in the United States, as the ST80 and ST30 clones are in Europe, Kuwait, Singapore, the Southwest Pacific islands and New Zealand (Udo et al. 2008), and Brazilian epidemic clone ST239 that is predominant in different hospitals (Carvalho et al. 2010, Almeida et al. 2012). Regarding S. epidermidis, the ST69 clone is predominant in Greece, ST57 and ST88 in Portugal, ST2 in China, ST2, ST22, ST61 and ST71 in Mexico and ST63 in Argentina (Miragaia et al. 2009, Liakopoulos et al. 2010).

Financial support: FAPEAM, Gestão Compartilhada em Saúde (PPSUS-014/2006)

CMF, FGN and WAF contributed equally to this work.

+ Corresponding author: cris_motta_ferr@yahoo.com.br

Received 13 March 2012

Accepted 12 September 2012
The risk for immunocompromised patients can be significant if they become infected by those species while in hospitals. The presence of immunocompromised patients at the hospital and blood bank where this research was undertaken was common, as the patients were receiving chemotherapy treatments against leukaemia, myeloma, haemolytic anaemia, haemophilia etc. These treatments can increase morbimortality risks due to prolonged hospitalisation, the spread of multi-resistant strains and rises in treatment costs. Given these clinically diagnosed infectious processes in immunocompromised patients, in this study, we studied the resistance of these pathogens to the antibiotics used in these treatments and the pathogens' genotypes for a broader epidemiological view of these staphylococci. Being aware of geographic distributions and transmission mechanisms - factors that contribute to the appearance and spread of these genotypes - will help us to control hospital infection outbreaks and dissemination and to implement programs to supervise the resistance presented by these pathogens.

From July 2007-August 2008, we conducted a study including haematology patients of both sexes admitted to the Haematology and Haemotherapy Foundation (HEMOAM) presenting clinical signs and symptoms suggestive of acute bacterial infection. The HEMOAM is located in Manaus, the capital city of the state of Amazonas. It is a public blood bank that provides health care to patients with haematological disorders and provides blood and blood derivatives to the residents of the entire city. The biological samples provided include urine, blood, sputum, catheter tips, bone marrow aspirate and abscess fluid, along with oropharyngeal, ocular, peria- 
nal, nasopharyngeal, open wound and skin lesion secretions. Phenotypic tests were performed on all isolates using standard biochemical tests, including Gram staining, colonial morphology on 5\% sheep's blood, mannitol and Mueller-Hinton agar plates (Himedia HexasystensMumbai, India), catalase, coagulase (Newprov), glucose, maltose, sucrose and lactose fermentation tests, urease (Himedia Hexasystens-Mumbai, India), susceptibility testing with $5 \mu \mathrm{g}$ of novobiocin (Laborclin) and $0.04 \mathrm{UI}$ bacitracin (Newprov), 300 polymyxin B (Newprov), PYR testing (Probac, Brazil) and nucleotide sequencing of the 16S rRNA gene region using the bacterial primers $27 \mathrm{~F}$ (5'-AGAGTTTGATCCTGGCTCAG-3') and 1492R (5'GGTTACCTTGTTACGACTT-3') (Miyoshi et al. 2005). The E-test ${ }^{\circledR}$ and reference values for sensitivity, reduced sensitivity or resistance to antibiotics were set according to the Clinical and Laboratory Standards Institute (CLSI 2010) (Wayne, Pennsylvania, USA) manual. ATCC 25923 S. aureus was used for quality control of the culture media and for susceptibility tests. PBP 2a tests (Oxoid, Cambridge, UK) were performed on all isolates to identify methicillin resistance. Genomic DNA extraction from all Staphylococcus strains was performed using an Kit Easy DNA (Invitrogen, Carlsbad, CA, USA) and a polymerase chain reaction (PCR) protocol for the identification of the mecA gene, SCCmec, toxin genes (seh, $\operatorname{arc} \mathrm{A}, e t d$ ) and the Panton-Valentine leukocidin gene, according to Jonas et al. (2002), Milheiriço et al. (2007) and Strommenger et al. (2008). S. aureus ATCC strains 25923, 29213 and 33591 and S. epidermidis ATCC 12228 were used as control strains. Multilocus sequence typing (MLST) was used to identify the genetic backgrounds of the isolates. MLST was performed according to the methods published for S. aureus and S. epidermidis. Internal fragments of the seven housekeeping genes were amplified by PCR and both strands were sequenced. Alleles and STs were determined from the S. aureus and S. epidermidis MLST databases (mlst.net). At the end of this research, the MLST website did not yet have proposal protocols for the genotypes of other coagulasenegative Staphylococcus species.

One hundred forty-six clinical samples were obtained from 69 patients over a period of 13 months. Among the collected samples, we were able to isolate 44 bacterial strains, 26 of which were Gram-positive. With regard to sex, 35 of 69 patients were male and 34 of 69 were female, with an average age of 24 years. The most frequent haematological diseases identified in patients were acute lymphocytic leukaemia (36/69) and acute myeloid leukaemia (6/69). Biochemical tests and 16S rDNA sequencing detected $S$. epidermidis (8/26), $S$. intermedius $(4 / 26)$ and $S$. aureus $(4 / 26)$. Other isolated species included Staphylococcus hyicus (1/26), S. haemolyticus (1/26), Staphylococcus simulans $(1 / 26)$, Staphylococcus lugdunensis (2/26) and Staphylococcus spp (5/26). Of the 26 staphylococci strains, 10 were methicillin-resistant. PBP 2a tests were performed on those 10 isolates, confirming the presence of the mecA gene and the absence of the lukPV gene. Only one sample of Staphylococcus sp. harboured the Seh gene for entero-

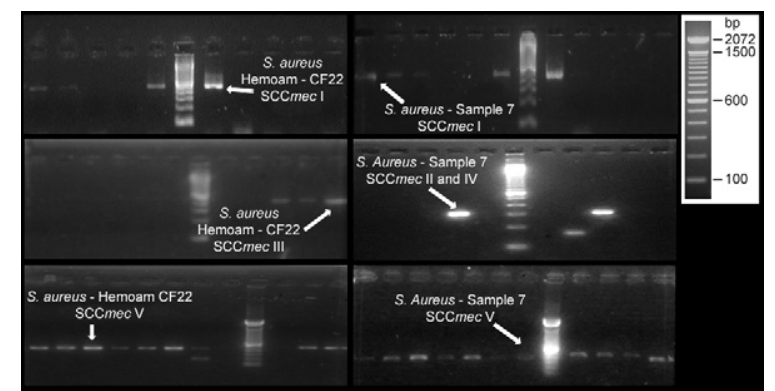

Polymerase chain reaction staphylococcal cassette chromosome mec (SCCmec) typing of Staphylococcus aureus strains. HEMOAM: Haematology and Haemotherapy Foundation.

toxin $\mathrm{H}$. The MLST analysis revealed three different STs among these isolates: $S$. aureus ST243, S. epidermidis ST2 and two new isolates of the S. epidermidis ST365 clone, which was not found among isolates in the MLST scheme of Miragaia et al. (2009) in our hospital environment. The ST243 strain harboured SCCmec types I, III and V (Figure), the ST2 strain harboured SCCmec types II, III and V and the ST365 strained harboured SCCmec types I, III and V (Table I). The other coagulasenegative Staphylococcus species were nontypeable. The DNA sequences of each allele at the seven loci in this study have been deposited in GenBank under accessions JN398483-JN398507. They can be downloaded from the aforementioned website.

The S. epidermidis ST 365 novel pathogenic clone was isolated from the peripheral blood of two patients: a boy ( 9 years old) and a girl ( 2 years old) presenting with acute lymphocytic leukaemia. Susceptibility tests indicated the resistance of these strains to antibiotics (Table II). The detection of this resistant methicillin isolate belonging to this pathogenic clonal lineage in hospitalised - mainly immunocompromised - patients can increase the risk for morbimortality if these patients acquire infections in hospitals caused by those species.

The $S$. epidermidis ST2 clone was isolated from a blood culture sample from a 24-year-old patient with chronic myeloid leukaemia presenting resistance to antibiotics, such as amikacin, ceftazidime and cefepime (Table II). The identification of these strains in the hospital might have been due to the following causes: (a) colonised patient (reservoir) circulation, (ii) contaminated healthcare worker-to-patient transmission, (iii) antibiotic selective pressure of the isolate (Sousa et al. 2009, Barbier et al. 2010), (iv) high biofilm formation - recognised as a critical factor for the colonisation of medical devices (Botelho et al. 2012), (v) high level of genetic flexibility including heterogeneous expression of the gene, (vi) the presence of SSCmec in diseaseassociated isolates, (vii) the ica gene cluster and (viii) insertion sequence element IS256. These causes reflect great capacity to adapt through genetic exchange to environmental changes and antibiotic therapy (Weisser et al. 2010, Widerström et al. 2012). 
TABLE I

Phenotype/genotype profiles of the methicillin-resistant staphylococcal strains isolated from patients with haematologic diseases

\begin{tabular}{|c|c|c|c|c|c|c|c|c|c|c|c|}
\hline \multirow[b]{2}{*}{ Strains } & \multirow{2}{*}{$\frac{\text { Phenotype }}{\text { PBP 2a }}$} & \multicolumn{10}{|c|}{ Genotype } \\
\hline & & $m e c \mathrm{~A}^{a}$ & $\mathrm{SCC} m e c$ & $\operatorname{arc} C$ & aroE & $g t R$ & mutS & $p y R$ & tpiA & $y g i L$ & ST \\
\hline $\begin{array}{l}\text { Staphylococcus epidermidis } \\
\text { (HEMOAM CF16 strain) }\end{array}$ & POS & POS & I, III, V & 3 & 25 & 5 & 5 & 3 & 4 & 4 & 365 \\
\hline $\begin{array}{l}\text { S. epidermidis } \\
\text { (HEMOAM CF36 strain) }\end{array}$ & POS & POS & I, III, V & 3 & 25 & 5 & 5 & 3 & 4 & 4 & 365 \\
\hline $\begin{array}{l}\text { S. epidermidis } \\
\text { (HEMOAM CF40 strain) }\end{array}$ & POS & POS & II, III, V & 7 & 1 & 2 & 2 & 4 & 1 & 1 & 2 \\
\hline $\begin{array}{l}\text { Staphylococcus aureus } \\
\text { (HEMOAM CF22 strain) }\end{array}$ & POS & POS & I, III, V & 2 & 2 & 5 & 2 & 6 & 3 & 2 & 243 \\
\hline
\end{tabular}

a: gold standard; HEMOAM: Haematology and Haemotherapy Foundation; POS: positive; SCCmec: staphylococcal cassette chromosome mec; ST: sequence type.

ST2, founder of the CC2 clonal complex, is considered the prevalent international cause of most $S$. epidermidis hospital infections; it has also been detected as an epidemic hospital pathogen worldwide and in different European hospitals (Weisser et al. 2010, Widerström et al. 2012). In Brazil, it was identified in a hospital in Rio de Janeiro (Iorio et al. 2012). The detection of methicillinresistant strains belonging to this virulent clonal lineage in hospitalised - mainly immunocompromised - patients points to possible hospital infections, as these patients had been hospitalised for more than $72 \mathrm{~h}$, they did not come from other hospitals, they received no prolonged antibiotic therapy at the moment of collection and they presented with frequent use of catheters because of their treatments. Therefore, studies monitoring the resistance of these strains to antibiotics are essential in light of the reports of coagulase-negative staphylococci species resistant to linezolid therapy, a therapeutic option for multidrug-resistant Gram-positive pathogens, including methicillin-resistant $S$. aureus and vancomycin-resistant enterococci (Almeida et al. 2012).

The $S$. aureus ST243 clone, isolated from a 30-yearold haemophilic patient with a blood infection, demonstrated resistance to antibiotics, such as ceftazidime, chloramphenicol and tetracycline (Table II). Despite in vitro sensitivity against imipenem and cefepime, CLSI M-100 S20 (CLSI 2010) recommendations suggest that they should be reported as resistant due to low treatment effectiveness. The presence of ST243 in our hospital could has come from patient-to-patient or colonised patient-to-contaminated healthcare worker transmission or from prolonged antibiotic therapy. Other risk factors that can be considered for haematogenous infection are nasal carriers and skin/soft tissue infections (Carvalho et al. 2010, Yamamoto et al. 2010, Widerström et al. 2012). This clone has spread across the United States, Thailand and Japan (data available from mlst.net), although only the Thai clone is methicillin-resistant.

The presence of the SCCmec III cassette in S. epidermidis, considered with no clinical importance in infec- tions must be revised (Tables I, III), because the horizontal transfer of a large number of resistance genes among species opens the way to methicillin resistance (Sousa et al. 2009, Barbier et al. 2010, Iorio et al. 2012), which induces the use of different antibiotics as therapeutic options (mainly vancomycin). Another observation was the presence of SCCmec type V in S. epidermidis (Table III). Types IV and V are small, structurally similar elements that carry the mecA gene as a unique antibiotic resistance encoder. As these types have high competitive transfer capacity, this fact can explain the presence of SCCmec type V in relation to type IV (Ito et al. 2004, Barbier et al. 2010). Another important factor detected was the existence of various SCCmec types (Figure, Tables I, III). Other Staphylococcus strains, such as $S$. intermedius and Staphylococcus sp., presented SCCmec types I, III and V. S.hyicus presented SCCmec types I, II and IV and S. lugdunensis SCCmec type III. The structural diversity of SCCmec in coagulase-negative Staphylococcus has been described in other studies (Ruppé et al. 2009 Barbier et al. 2010, Carvalho et al. 2010). The presence of these different $\mathrm{SCCmec}$ types might suggest that multiple introductions are occurring and their presence in the same ST could suggest possible horizontal transfer among species (Enright et al. 2000, Barbier et al. 2010) or diversity in the combination of the mec and $c c r$ allotypes (Ruppé et al. 2009).

Horizontal transfer of the mec gene might be occurring, favouring the appearance of methicillin resistance among species. These findings serve as an alert for hospitals and possibly for the region to introduce actions to reduce the spread of the pathogenic clonal lineages of $S$. aureus and S. epidermidis. Microbial resistance monitoring programs are important to avoid hospital infection outbreaks and the spread of pathogenic clonal lines. Further research in regional hospitals and the community are required for a more accurate view of the regional epidemiological distribution of these clones and of the new ST365 clone. 


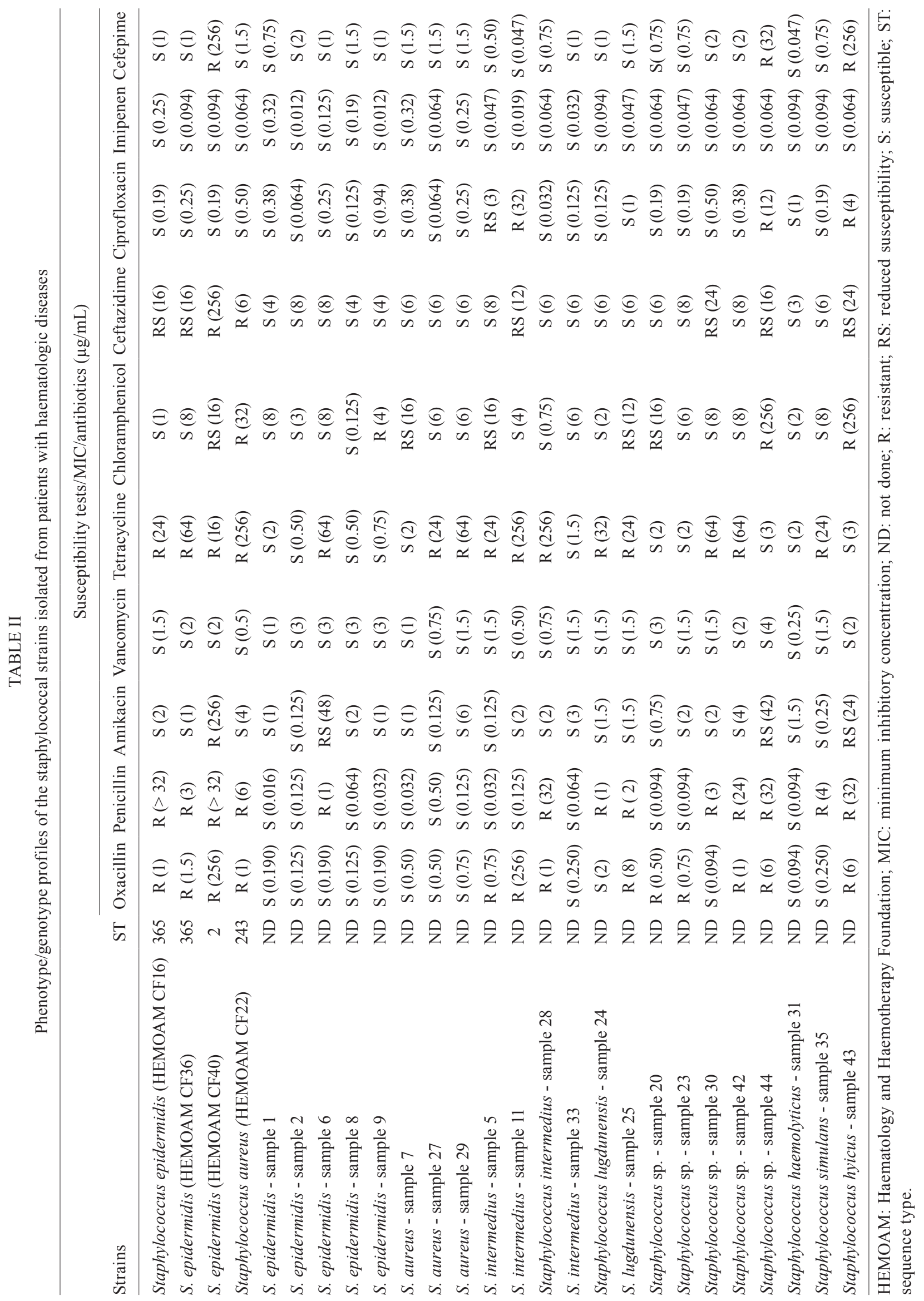


TABLE III

Phenotype/genotype profile of the staphylococcal species isolated from patients with haematologic diseases

\begin{tabular}{|c|c|c|c|}
\hline \multirow[b]{2}{*}{ Strains } & \multicolumn{2}{|c|}{ Phenotype } & \multirow{2}{*}{$\begin{array}{l}\text { Genotype } \\
\text { SCCmec }\end{array}$} \\
\hline & PBP 2a & $m e c \mathrm{~A}^{a}$ & \\
\hline Staphylococcus aureus - sample 7 & NEG & NEG & $\mathrm{I}, \mathrm{II}$ or $\mathrm{IV}^{b}, \mathrm{~V}$ \\
\hline Staphylococcus intermedius - sample 28 & POS & POS & I, III, V \\
\hline Staphylococcus sp. - sample 20 & POS & POS & I, III, V \\
\hline Staphylococcus sp. - sample 23 & NEG & POS & III, V \\
\hline Staphylococcus epidermidis - sample 8 & NEG & NEG & $\mathrm{I}$ \\
\hline S. epidermidis - sample 9 & NEG & NEG & I \\
\hline Staphylococcus sp. - sample 42 & NEG & NEG & I \\
\hline Staphylococcus sp. - sample 44 & POS & POS & I \\
\hline Staphylococcus hyicus- sample 43 & POS & POS & $\mathrm{I}, \mathrm{II}$ or $\mathrm{IV}^{b}$ \\
\hline Staphylococcus lugdunensis - sample 24 & POS & POS & III \\
\hline S. epidermidis - sample 2 & NEG & NEG & NT \\
\hline S. epidermidis - sample 6 & NEG & NEG & NT \\
\hline S. aureus - sample 27 & NEG & NEG & NT \\
\hline S. aureus - sample 29 & NEG & NEG & NT \\
\hline S. intermedius - sample 5 & NEG & NEG & NT \\
\hline S. intermedius - sample 11 & NEG & NEG & NT \\
\hline S. intermedius - sample 33 & NEG & NEG & NT \\
\hline S. epidermidis - sample 1 & NEG & NEG & NT \\
\hline S. lugdunensis - sample 25 & NEG & NEG & NT \\
\hline Staphylococcus sp. - sample 30 & NEG & NEG & NT \\
\hline Staphylococcus haemolyticus - sample 31 & NEG & NEG & NT \\
\hline Staphylococcus simulans - sample 35 & NEG & NEG & NT \\
\hline
\end{tabular}

$a$ : gold standard; $b$ : primers amplifies both staphylococcal cassette chromosome mec (SCCmec) II or IV; NEG: negative; NT: nontypeable; POS: positive.

\section{REFERENCES}

Almeida LM, Lincopan N, Mamizuka EM 2012. Clonal dissemination of linezolid-resistant Staphylococcus haemolyticus exhibiting the G2576T mutation in the 23S rRNA gene in a tertiary care hospital in Brazil. Antimicrob Agents Chemother 56: 27922793.

Barbier F, Ruppé E, Hernandez D, Lebeaux D, Francois P, Felix B, Desprez A, Maiga A, Woerther PL, Gaillard K, Jeanrot C, Wolff M, Schrenzel J, Adremont A, Ruimy R 2010. Methicillin-resistant coagulase-negative staphylococci in the community: high homology of SCCmec IVa between Staphylococcus epidermidis and major clones of methicillin-resistant Staphylococcus aureus. J Infect Dis 202: 270-281.

Botelho AMS, Nunes ZG, Asensi MD, Gomes MZR, Fracalanzza SE, Figueiredo AMS 2012. Characterization of coagulase-negative staphylococci isolated from hospital indoor air and a comparative analysis between airborne and inpatient isolates of Staphylococcus epidermidis. J Med Microbiol 61: 1136-1145.

Carvalho KS, Mamizuka EM, Gontijo Filho PP 2010. Methicillin/oxacillin-resistant Staphylococcus aureus as a hospital and public health threat in Brazil. Braz J Infect Dis 14: 71-76.

CLSI - Clinical and Laboratory Standards Institute 2010. Performance standards for antimicrobial susceptibility testing, 20th Information Suplement M100-S20, CLSI, Wayne, 156 pp.
Enright MC, Nicholas PJD, Davies CE, Peacock SJ, Spratt B 2000. Multilocus sequence typing for characterization of methicillin - resistant and methicillin-susceptible clones of Staphylococcus aureus. J Clin Microbiol 38: 1008-1015.

Iorio NLP, Caboclo RF, Azevedo MB, Barcellos AG, Neves FPG, Domingues RMCP, dos Santos KRN 2012. Characteristics related to antimicrobial resistance and biofilm formation of widespread methicillin-resistant Staphylococcus epidermidis ST2 and ST23 lineages in Rio de Janeiro hospitals, Brazil. Diagn Microbiol Infect Dis 72: 32-40.

Ito T, Ma XX, Takeuchi F, Okuma K, Yuzawa H, Hiramatsu K 2004. Novel type V staphylococcal cassette chromosome mec driven by a novel cassette chromosome recombinase, $c c r C$. Antimicrob Agents Chemother 48: 2637-2651.

Jonas D, Speck M, Daschner FD, Grundmann H 2002. Rapide PCRbased identification of methicillin - resistant Staphylococcus aureus from screening swabs. J Clin Microbiol 40: 1821-1823.

Liakopoulos A, Spiliopoulou I, Damani A, Kanellopoulou M, Schoina S, Papafragas E, Marangos M, Fligou F, Zakynthinos E, Makris D, Protonotariou E, Tsiapara F, Filos J, Diza E, Anastassiou D, Petinaki E 2010. Dissemination of two international linezolidresistant Staphylococcus epidermidis clones in Greek hospitals. J Antimicrob Chemother 65: 1070-1077.

Milheiriço C, Oliveira DC, de Lencastre H 2007. Update to the multiplex strategy for assignment of mec element types in Staphylococcus aureus. Antimicrob Agents Chemother 51: 3374-3377. 
Miragaia M, Lencastre H, Perdreau-Remington F, Chambers HF, Higashi J, Sullam PM, Lin J, Wong KI, King KA, Otto M, Sensabaugh GF, Diep BA 2009. Genetic diversity of arginine catabolic mobile element in staphylococcus epidermidis. PLoS ONE 4: e7722.

Miyoshi T, Iwatsuki T, Naganuma T 2005. Phylogenetic characterization of 16S rRNA gene clones from deep-groundwater microorganisms that pass through 0.2-micrometer-pore-size filters. Appl Environ Microbiol 71: 1084-1088.

Ruppé E, Barbier F, Mesli Y, Maiga A, Cojocaru R, Benkhalfat M, Benchouk S, Hassaine H, Maiga I, Diallo A, Nareth C, Sarthou JL, Andremont A, Ruimy R 2009. Diversity of staphylococcal cassete chromosome mec structures in methicillin-resistant Staphylococcus epidermidis and Staphylococcus haemolyticus strains among outpatients from four countries. Antimicrob Agents Chemother 53: 442-449.

Sousa FCJ, Nunes EWF, Nascimento ED, Oliveira SM, Melo MCN, Fernandes MJBC 2009. Prevalência de Staphylococcus spp resistentes à meticilina isolados em uma maternidade escola da cidade de Natal, estado do Rio Grande do Norte. Rev Soc Bras Med Trop 42: 179-182.

Strandén AM, Frei R, Adler H, Flückiger U, Widmer AF 2009. Emergence of SCCmec type IV as the most common type of meth- icillin-resistant Staphylococcus aureus in a university hospital. Infection 37: 44-48.

Strommenger B, Braulke C, Pasemann B, Schmidt C, Witte W 2008. Multiplex PCR for rapid detection of Staphylococcus aureus isolates suspected to represent community acquired strains. J Clin Microbiol 46: 582-587.

Udo EE, O'Brien FG, Al-Sweih N, Noronha B, Matthew B, Grubb WB 2008. Genetic lineages of community-associated methicillin-resistant Staphylococcus aureus in Kuwait hospitals. J Clin Microbiol 46: 3514-3516.

Weisser M, Schoenfelder SMK, Orasch C, Arber C, Gratwohl A, Frei R, Eckart M, Fluckiger U, Ziebuhr W 2010. Hypervariability of biofilm formation and oxacillin resistance in a Staphylococcus epidermidis strain causing persistent severe infection in an immunocompromised patient. J Clin Microbiol 48: 2407-2412.

Widerström M, Wiström J, Sjöstedt A, Monsen T 2012. Coagulasenegative staphylococci: update on the molecular epidemiology and clinical presentation with a focus on Staphylococcus epidermidis and Staphylococcus saprophyticus. Eur J Clin Microbiol 31: 7-20.

Yamamoto T, Nishiyama A, Takano T, Yabe S, Higuchi W, Razvina O, Shi D 2010. Community-acquired methicillin-resistant Staphylococcus aureus: community transmission, pathogenesis and drug resistance. J Infect Chemother 16: 225-254. 